\title{
Prognostic impact of baseline and residual SYNTAX scores in cardiogenic shock
}

\section{Javanainen, Tuija}

2019-01-01

Javanainen , T , Sans-Rosello , J , Harjola , V-P , Nieminen , M S , Lassus , J , Sionis , A , Varpula, M \& Jurkko, R 2019 , ' Prognostic impact of baseline and residual SYNTAX scores in cardiogenic shock ' , Catheterization and Cardiovascular Interventions , vol. 93 , no. 1 , pp. 1-8 . https://doi.org/10.1002/ccd.27716

http://hdl.handle.net/10138/312517

https://doi.org/10.1002/ccd.27716

publishedVersion

Downloaded from Helda, University of Helsinki institutional repository.

This is an electronic reprint of the original article.

This reprint may differ from the original in pagination and typographic detail.

Please cite the original version. 


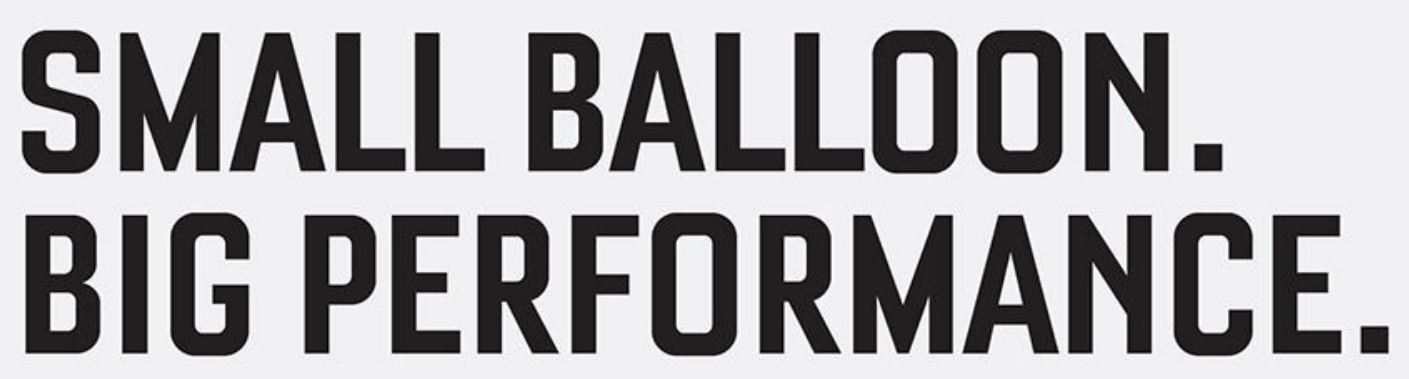

THE 1ST AND ONLY 1.0 MM BALLOON WITH FDA CLEARANCE.

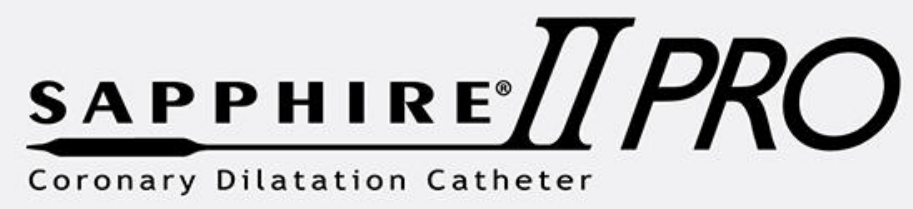

\section{That's the power of constant progress.}

Distributed by

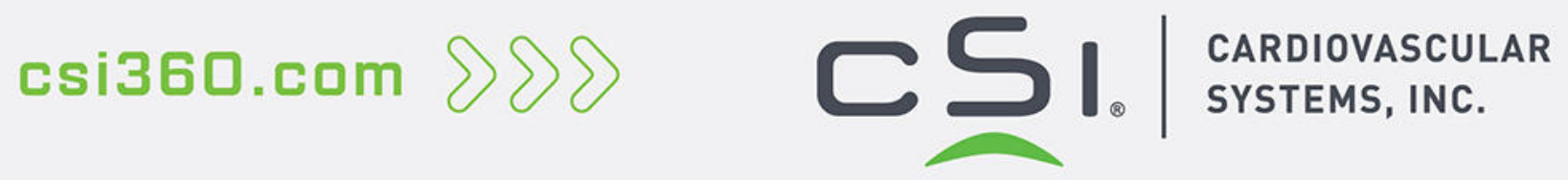




\title{
Prognostic impact of baseline and residual SYNTAX scores in cardiogenic shock
}

\author{
Tuija Javanainen MD ${ }^{1}$ (1) | Jordi Sans-Roselló MD $^{2}$ | Veli-Pekka Harjola Assoc. Prof. ${ }^{3}$ | \\ Markku S. Nieminen Prof. ${ }^{1}$ | Johan Lassus Assoc. Prof. ${ }^{1}$ | Alessandro Sionis Assoc. Prof. ${ }^{2}$ | \\ Marjut Varpula $\mathrm{PhD}^{1}$ | Raija Jurkko $\mathrm{PhD}^{1}$
}

${ }^{1}$ Cardiology, University of Helsinki, Heart and Lung Center, Helsinki University Hospital, Helsinki, Finland

${ }^{2}$ Department of Cardiology, Acute and Intensive Cardiovascular Care Unit, Hospital de la Santa Creu i Sant Pau, Biomedical Research Institute IIB-Sant Pau, Universitat Autònoma de Barcelona, CIBER-CV, Barcelona, Spain

${ }^{3}$ Emergency Medicine, Department of Emergency Medicine and Services, University of Helsinki, Helsinki University Hospital, Helsinki, Finland

\section{Correspondence}

Tuija Javanainen, MD, Tallbergin puistotie 7B26, 00200 Helsinki, Finland.

Email: tuija.javanainen@outlook.com

Funding information

Finnish Cardiac Society, ACS and secondary prevention grant, supported by AstraZeneca; Paavo Nurmen Säätiö

\begin{abstract}
Objectives: The aim was to assess the extent of coronary artery disease and revascularization using baseline SYNTAX Score (bSS) and residual SYNTAX Score (rSS) in patients with cardiogenic shock (CS) secondary to ST-segment elevation myocardial infarction (STEMI). The prognostic impact of SYNTAX Score (SS) was evaluated and assessed for additive value over clinical risk scores.

Background: bSS and rSS have been proven to be useful in risk stratification in stable coronary artery disease as well as in acute coronary syndromes, but they have not been studied in STEMI related CS.

Methods: Patients from a multinational prospective study of CS were analyzed. The study population was divided into tertiles according to bSS. The Cox regression and receiver operating characteristic (ROC) curves were used to assess the predictive power of SS.

Results: Of the 61 studied patients, $85 \%$ were male and the mean age was 67 years. Median bSS was 22 (15-32) and rSS 7 (0-13). Ninety-day mortality was 43\%. bSS had negative prognostic value in multivariable analysis (HR 1.06, 95\% Cl 1.01-1.10). However, additive value over clinical risk scores was limited. rSS was not associated with mortality, whereas postpercutaneous coronary intervention (PCI) TIMI flow 3 of infarct-related artery (IRA) predicted better survival.

Conclusions: In STEMI related CS, the added value of bSS and rSS over clinical assessment and risk scores is limited. Our results suggest that while immediate $\mathrm{PCl}$ in order to restore blood flow to the IRA is essential, deferring the treatment of residual lesions does not seem to be associated with worse prognosis.
\end{abstract}

KEYWORDS

acute coronary syndrome, prognosis, revascularization, shock

\section{1 | INTRODUCTION}

SYNTAX Score (SS) is an angiographic scoring system that measures the burden of coronary artery disease and has been shown to be an independent predictor of cardiovascular events and mortality in stable coronary artery disease ${ }^{1}$ as well as in acute coronary syndromes (ACS). ${ }^{2-6}$ In the current literature, there are no data describing the prognostic value of baseline SS (bSS) in ST-segment elevation myocardial infarction (STEMI) related cardiogenic shock (CS). Residual SS (rSS) measures the extent of coronary disease after percutaneous coronary intervention ( $\mathrm{PCl}$ ) and can be used as an indicator of completeness of revascularization. Current guidelines suggest multivessel $\mathrm{PCl}$ in $\mathrm{CS}^{7}$ but new data about revascularization strategies in $\mathrm{CS}$ have been published after these recommendations: the CULPRIT-SHOCK trial showed worse outcome in CS with multivessel $\mathrm{PCl}$ in comparison with revascularization of culprit artery only. ${ }^{8}$ Previously, the predictive 
value of rSS has been shown in stable coronary artery disease, ${ }^{9-11}$ as well as in patients with $\mathrm{ACS},{ }^{12-14}$ but not studied in the context of CS.

The aim of this study was to evaluate the extent of coronary artery disease and success of revascularization in STEMI-related CS patients treated with $\mathrm{PCl}$. Second, the predictive value of bSS and rSS in CS patients was evaluated over clinical and angiographic values, including TIMI flow grade of the infarct-related artery (IRA). Finally, we assessed the additive prognostic value of SS over three clinical risk scores: CardShock risk score, ${ }^{15}$ IABP-SHOCK II risk score ${ }^{16}$ and GRACE risk score. ${ }^{17}$

\section{2 | MATERIALS AND METHODS}

\section{1 | Study population}

This is a sub-analysis of the CardShock study, a prospective observational multicenter investigation of the prognosis and treatment of CS. The CardShock study was conducted from October 2010 to December 2012 in eight European countries in nine hospitals. The design, methodology and primary results have been published before. $^{15}$ The study was approved by local ethics committees and conducted in accordance with the Declaration of Helsinki. Written informed consent was obtained from each patient or a next of kin.

Briefly, adult patients who fulfilled predefined criteria for CS were included within $6 \mathrm{hr}$ from the detection of CS. The criteria for shock were systolic blood pressure less than $90 \mathrm{mmHg}$ for at least 30 minutes despite adequate fluid therapy or need for vasoactive therapy, and $\geq 1$ signs of inadequate organ perfusion: confusion or altered mental status, cool extremities, oliguria less than $0.5 \mathrm{ml} / \mathrm{kg} / \mathrm{h}$ for the previous $6 \mathrm{hr}$, or blood lactate greater than $2 \mathrm{mmol} / \mathrm{l}$. Patients with ongoing hemodynamically significant arrhythmia, or shock after cardiac or non-cardiac surgery were excluded.

Patients' demographics, medical history and clinical characteristics were collected. Hemodynamic parameters and laboratory measurements were registered. Arterial blood lactate and $\mathrm{pH}$ were analyzed locally and creatinine, high-sensitivity troponin $\mathrm{T}$ (hs-TnT), and $\mathrm{N}$ terminal pro-B-type natriuretic peptide (NT-proBNP) (Roche Diagnostics, Basel, Switzerland) were analyzed centrally. Estimated glomerular filtration rate (eGFR) was calculated from creatinine values using the CKD-EPI Creatinine equation. Echocardiography was performed at study entry. The patients were treated according to local practice and given treatments were registered.

For the present substudy, angiographic images from the two largest centers (Helsinki and Barcelona) were analyzed and combined with the clinical data from the CardShock database. STEMI patients treated with primary $\mathrm{PCl}$ or rescue $\mathrm{PCl}$ were included. STEMI was defined according to the third universal definition of myocardial infarction. ${ }^{18}$ Exclusion criteria were previous coronary artery bypass grafting (CABG), emergency CABG after angiography, or mechanical complication of STEMI as an etiology of CS. The primary endpoint was allcause mortality at 90-day follow-up. The vital status was confirmed by the patient or the next of kin, or through hospital or population registers.

\section{2 | SYNTAX scores}

SS was measured from angiographic images using the SS algorithm. ${ }^{19}$ Two experienced cardiologists (MV and JS) analyzed the angiograms post hoc blinded to patient data. The interobserver variability (tertial partitioning) for the SS examiners was calculated in 48 independently analyzed angiograms: Cohen's Kappa statistics was $0.59(95 \% \mathrm{Cl} 0.40-0.78, P<0.01)$ for bSS and $0.65(95 \% \mathrm{Cl}$ 0.32-0.97, $P<0.001$ ) for $\mathrm{rSS}$, signifying moderate interobserver reliability comparable to the SYNTAX trial ${ }^{1}$ and also to the previous studies with ACS patients. ${ }^{3,5,21}$

All lesions with stenosis greater than $50 \%$ in vessels with diameter of more than $1.5 \mathrm{~mm}$ were scored. SS was calculated at three time points:

1. Baseline SS1 (bSS1) was measured from initial diagnostic angiogram. Completely occluded IRA with TIMI flow 0 or 1 was scored as a total occlusion.

2. Baseline SS2 (bSS2) was measured after wiring or thrombectomy, if TIMI flow of IRA improved and severity of the lesions could be measured downstream. If TIMI flow did not improve or anatomy of IRA could not be assessed, bSS2 was the same as bSS1.

3. Residual SS was measured after primary $\mathrm{PCl}$ was completed. If further angiograms were performed during the hospital stay, the rSS was measured after all $\mathrm{PCl}$ procedures.

\section{3 | Statistical analysis}

The continuous data are presented as means and standard deviations (SD) or as medians and interquartile ranges (IQR). The study population was divided into tertiles by bSS1. Differences between tertiles were analyzed with one-way ANOVA, the Kruskal-Wallis or logistic regression as appropriate. The changes in SS were compared with Wilcoxon's Signed-Rank test. The Kaplan-Meier method was used to generate survival curves and log-rank test was used to assess differences in survival. The prognostic evaluation was also conducted in different rSS cutoffs $(0,8$, and 12 points), defined in previous literature. ${ }^{12,20,21}$ Receiver operating characteristic (ROC) curves were constructed to assess the predictive power of bSS and rSS for 90-day mortality. The association with mortality was assessed for relevant control variables (age, gender, hypertension, diabetes, coronary artery disease, previous myocardial infarction, previous $\mathrm{PCl}$, chronic heart failure, resuscitation, systolic blood pressure, left ventricular ejection fraction (LVEF), eGFR, hs-TnT, NT-proBNP, arterial blood lactate, $\mathrm{pH}$, one-vessel disease, three-vessel disease, left main disease, chronic total occlusion (CTO), acute thrombus, multivessel $\mathrm{PCl}$, drug-eluting stent, prior PCI TIMI flow 0 , and post-PCI TIMI flow 3) by the univariate Cox regression and variables with $P$-value less than 0.05 were selected for multivariable analysis. The final multivariable model included age, LVEF, arterial blood lactate, eGFR, NT-proBNP, and post-PCI TIMI flow 3 and the model was built in a forward stepwise method with the Cox proportional analysis. In addition, three multivariable models were created to evaluate the additive prognostic value of bSS and rSS over clinical risk scores: bSS or rSS and either CardShock risk score, ${ }^{15}$ IABP-SHOCK II risk score ${ }^{16}$ or GRACE risk score. ${ }^{17}$ An 
interaction analysis between CS timing (before or after revascularization) and rSS was also conducted. A two-tailed $P$-value of less than 0.05 was considered statistically significant. Statistical analyses were performed using SPSS 24.0.0.0 statistical software (IBM Corp, Armonk, NY, USA).

\section{3 | RESULTS}

\subsection{Baseline characteristics and SS results}

The patient selection process $(n=61)$ for this substudy is described in Figure 1. Briefly, mean age was $67( \pm 12)$ years, $85 \%$ were male. Median bSS1 at baseline was 22 (15-32). After wiring or thrombectomy, median bSS2 was 19 (11-30), significantly lower than bSS1 $(P<0.01)$. After revascularization, median rSS was $7(0-13)$, lower than bSS1 and bSS2 (both $P<0.01$ ). Only two patients had second angiography after primary $\mathrm{PCl}$, but the angiogram for second procedure was available for assessment for one patient only.

Table 1 summarizes baseline characteristics according to bSS tertiles (the first tertile: bSS1 $\leq 18$; the second tertile: bSS1 19-27; the third tertile: bSS1 > 27). There were no differences among the tertiles except for history of previous $\mathrm{PCl}$ that was lower in the first tertile in comparison to higher tertiles (both $P<0.01$ ). There were no differences in hemodynamic parameters and routine laboratory measurements (Table 1).

\section{2 | Angiographic findings}

One-third of the patients had one-vessel disease, 39\% had two-vessel disease, and $23 \%$ three-vessel disease. In the first and the second bSS tertiles, only one patient $(5 \%)$ in each group had CTO, while in the third tertile $75 \%$ had CTO (both $P<0.01$ ). Over half of the patients (64\%) had acute occlusive thrombosis of IRA and there were no differences between tertiles $(P=0.45)$. Left main was affected approximately in $15 \%$ in all tertiles $(P=0.99)$ (Table 1$)$.

Multivessel $\mathrm{PCl}$ was performed with similar frequency in all bSS tertiles (28\%) and the use of drug-eluting stents (33\%) did not differ between the groups. Complete revascularization ( $\mathrm{rSS} 0$ ) was achieved in $28 \%$ of the patients. rSS was lower in the first and the second tertiles in comparison with the third tertile (both $P<0.01$ ). More than

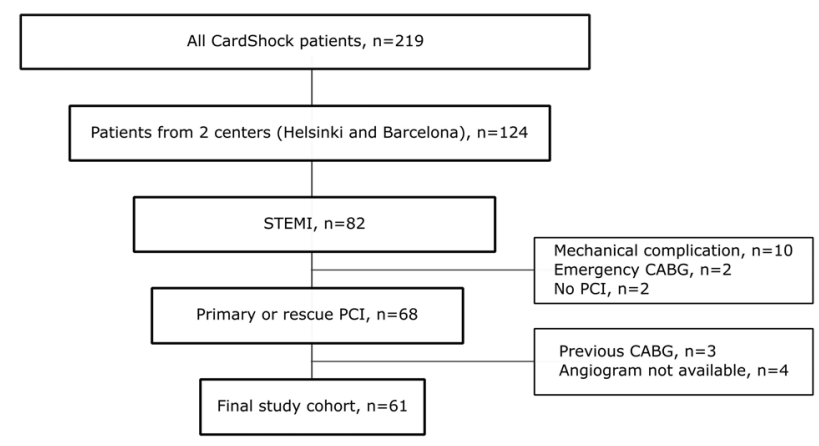

FIGURE 1 Patient flowchart-STEMI patients treated with primary $\mathrm{PCl}$ or rescue $\mathrm{PCl}$ were included in this substudy. Exclusion criteria were previous CABG, emergency CABG after angiography, or mechanical complication of STEMI as an etiology of CS half of the patients ( $\mathrm{n}=36,59 \%$ ) had shock before $\mathrm{PCl}$ (Table 1 ) but there was no difference in either SS whether the patient had shock before or after the procedure (Supporting Information Table 1). At discharge, $94 \%$ of the patients were on dual antiplatelet therapy (Table 1).

\section{3 | Mortality}

All-cause 90 -day mortality was $43 \%$. Mortality rate was lower in the first tertile (19\%), in comparison with the second $(50 \%)$ and the third tertiles (60\%) (Figure 2A). In univariate analysis, bSS predicted 90-day mortality (HR 1.04, 95\% Cl 1.01-1.08) and the association was independent after adjustment for control variables (HR $1.06,95 \% \mathrm{Cl}$ 1.01-1.10). bSS predicted mortality also after adjustment with the IABP-SHOCK II (HR 1.05, 95\% Cl 1.01-1.09) and GRACE (HR 1.04, 95\% $\mathrm{Cl} 1.00-1.08)$ risk scores but not when adjusted for the CardShock risk score (HR 1.02, 95\% Cl 0.98-1.06) (Table 2A).

There were no statistically significant differences in mortality when using different rSS cutoff points (0, 8, and 12) (Figure 2B,D). In univariate analysis, rSS as a continuous variable correlated with 90-day mortality (HR 1.04, 95\% Cl 1.00-1.07), but not independently in any of the multivariable models (Table $2 \mathrm{~A}$ ). rSS as a categorical variable did not associate with mortality in univariate or multivariable analysis (Table $3 \mathrm{~A}$ ). There was no interaction between the timing of CS (before or after coronary angiography) and rSS. Figure 3 depicts the ROC-curves and AUCs for the bSS and rSS in comparison with the three risk scores.

\section{4 | DISCUSSION}

To the best of our knowledge, this is the first study that evaluates the prognostic value of bSS and rSS in patients with STEMI-related CS. We found distinctive improvement from bSS to rSS implying successful revascularization. bSS seems to associate with short-term mortality, but the additive value over pre-existing clinical risk scores is limited. The prognostic effect of rSS could not be shown, but post- $\mathrm{PCI}$ TIMI flow 3 of IRA predicted better survival.

The median bSS in this study was lower than in the original SYNTAX study, ${ }^{22}$ but higher than in previous studies concerning STEMI. ${ }^{2,5,6,12}$ The median bSS was higher than described in another CS population, ${ }^{23}$ yet the definition of CS in our study was stricter. Most of the patients in this study had multivessel disease and one fourth had CTO, indicating that the patients with STEMI-related CS have pre-existing complex coronary artery lesions. Regarding the risk of CS in STEMI patients, CS has been more prevalent in the patients with high $\mathrm{SS}^{5,6}$ and $\mathrm{SS}$ has been identified as an independent predictor of CS in ACS patients. ${ }^{23}$ The results are in line with our finding of high bSS in STEMI-related CS.

The prognostic value of bSS in STEMI-related CS was evaluated for the first time. bSS retained prognostic value in CS but its clinical relevance was limited, especially when added to recently developed risk scores for $\mathrm{CS}$. The result differs to some extent from observations in non-shock STEMI patients in which bSS was credited with a robust predictive value. ${ }^{2,4-6,10}$ However, in these STEMI populations, the 
TABLE 1 Baseline characteristics, SYNTAX scores, clinical data, and mortality in bSS1 tertiles

\begin{tabular}{|c|c|c|c|c|}
\hline & $\begin{array}{l}\text { First tertile bSS1 } \leq 18 \\
n=21\end{array}$ & $\begin{array}{l}\text { Second tertile bSS1 19-27 } \\
n=20\end{array}$ & $\begin{array}{l}\text { Third tertile bSS }>27 \\
n=20\end{array}$ & $P$-value \\
\hline Age, years (SD) & $62( \pm 12)$ & $68( \pm 13)$ & $70( \pm 12)$ & 0.92 \\
\hline Male gender, $n(\%)$ & $20(95 \%)$ & $14(70 \%)$ & $18(90 \%)$ & 0.06 \\
\hline Resuscitation, $n$ (\%) & 9 (43\%) & 7 (35\%) & $3(15 \%)$ & 0.12 \\
\hline \multicolumn{5}{|l|}{ SYNTAX scores } \\
\hline bSS2, pts (IQR) & $7(4-11)$ & $13(12-16)$ & $32(22-36)$ & $<0.01$ \\
\hline \multicolumn{5}{|l|}{ Medical history } \\
\hline Coronary artery disease, $n$ (\%) & $2(10 \%)$ & $4(20 \%)$ & $7(35 \%)$ & 0.13 \\
\hline Previous $\mathrm{PCl}, n$ (\%) & $0(0 \%)$ & $3(15 \%)$ & $6(30 \%)$ & $<0.01$ \\
\hline Diabetes, $n(\%)$ & $5(24 \%)$ & $2(10 \%)$ & $8(40 \%)$ & 0.08 \\
\hline Hypertension, n (\%) & $11(52 \%)$ & $11(55 \%)$ & $13(65 \%)$ & 0.69 \\
\hline LVEF, \% (SD) & $37( \pm 15)$ & $34( \pm 14)$ & $29( \pm 9)$ & 0.17 \\
\hline SBP, mmHg (SD) & $77( \pm 23)$ & $77( \pm 11)$ & $76( \pm 12)$ & 0.98 \\
\hline Serum lactate, mmol/I (IQR) & $2.2(1.2-3.2)$ & $2.3(1.7-6.3)$ & $2.8(2.1-4.4)$ & 0.31 \\
\hline Creatinine, $\mu \mathrm{mol} / \mathrm{I}(\mathrm{IQR})$ & $89(72-110)$ & $97(74-116)$ & $112(85-136)$ & 0.20 \\
\hline eGFR, ml/min (IQR) & $88(65-104)$ & $78(42-98)$ & $67(41-87)$ & 0.15 \\
\hline Hs-TnT, ng/l (IQR) & $2,427(849-6,810)$ & $7,236(3,677-11,943)$ & $2,889(1,828-8,965)$ & 0.04 \\
\hline NT-proBNP, ng/l (IQR) & $198(133-942)$ & $1,471(253-3,977)$ & $3,914(481-16,551)$ & $<0.01$ \\
\hline \multicolumn{5}{|l|}{ Angiographic data } \\
\hline Acute occlusive thrombosis, $n(\%)$ & $12(57 \%)$ & $15(75 \%)$ & $12(60 \%)$ & 0.45 \\
\hline Time from angiography to shock, min (IQR) & $180(60-300)$ & $20(15-75)$ & $98(45-175)$ & 0.47 \\
\hline Second PCI, $n$ (\%) & $0(0 \%)$ & $1(5.0 \%)$ & $1(5 \%)$ & 0.41 \\
\hline \multicolumn{5}{|l|}{ Treatment } \\
\hline Multivessel PCl, $n$ (\%) & $4(19 \%)$ & $6(30 \%)$ & $7(35 \%)$ & 0.50 \\
\hline Drug-eluting stent, $n$ (\%) & $8(38 \%)$ & $6(30 \%)$ & $6(30 \%)$ & 0.82 \\
\hline Complete revascularization (rSS 0), $n$ (\%) & $12(57 \%)$ & $5(25 \%)$ & $0(0 \%)$ & $<0.01$ \\
\hline Post-PCI TIMI 3, n (\%) & $14(67 \%)$ & $12(60 \%)$ & 14 (74\%) & 0.66 \\
\hline IABP, $n(\%)$ & $10(48 \%)$ & 14 (70\%) & $13(65 \%)$ & 0.31 \\
\hline \multicolumn{5}{|l|}{ Medication at discharge $e^{\mathrm{a}}$} \\
\hline Aspirin $^{\mathrm{a}}, n(\%)$ & $16(94 \%)$ & $11(100 \%)$ & 8 (100\%) & 0.47 \\
\hline Clopidogrel/prasugel $^{\mathrm{a}}, n$ (\%) & 15 (88\%) & $11(100 \%)$ & $7(88 \%)$ & 0.22 \\
\hline \multicolumn{5}{|l|}{ Endpoints } \\
\hline 90-Day mortality, n (\%) & $4(19 \%)$ & $10(50 \%)$ & $12(60 \%)$ & 0.02 \\
\hline
\end{tabular}

Abbreviations: bSS, baseline SYNTAX Score; CS, cardiogenic shock; eGFR, estimated glomerular infiltration; hs-TnT, high-sensitivity troponin T; IABP, intra-aortic balloon pump; LVEF, left ventricular ejection fraction; MACCE, major adverse cardiac and cerebrovascular events; NT-proBNP, N-terminal pro-b-type natriuretic peptide; $\mathrm{PCl}$, percutaneous coronary intervention; rSS, residual SYNTAX Score; SBP, systolic blood pressure. Data are presented as numbers (\%), means (SD) and medians (IQR).

${ }^{a}$ Percentages calculated in patients who were discharged $(n=36)$.

prevalence of CS was very low, which could explain the difference in outcomes. In the present study, bSS had some additive predictive value over the GRACE and IABP-SHOCK II risk scores. Previously, in a non-CS STEMI population, bSS was shown to be useful in risk stratification over GRACE risk score. ${ }^{24}$ Nevertheless, we believe, that in the acute clinical context of CS, using clinical risk scores for risk stratification is more feasible and accurate than assessing the exact burden of coronary artery disease with bSS. However, the sample size 

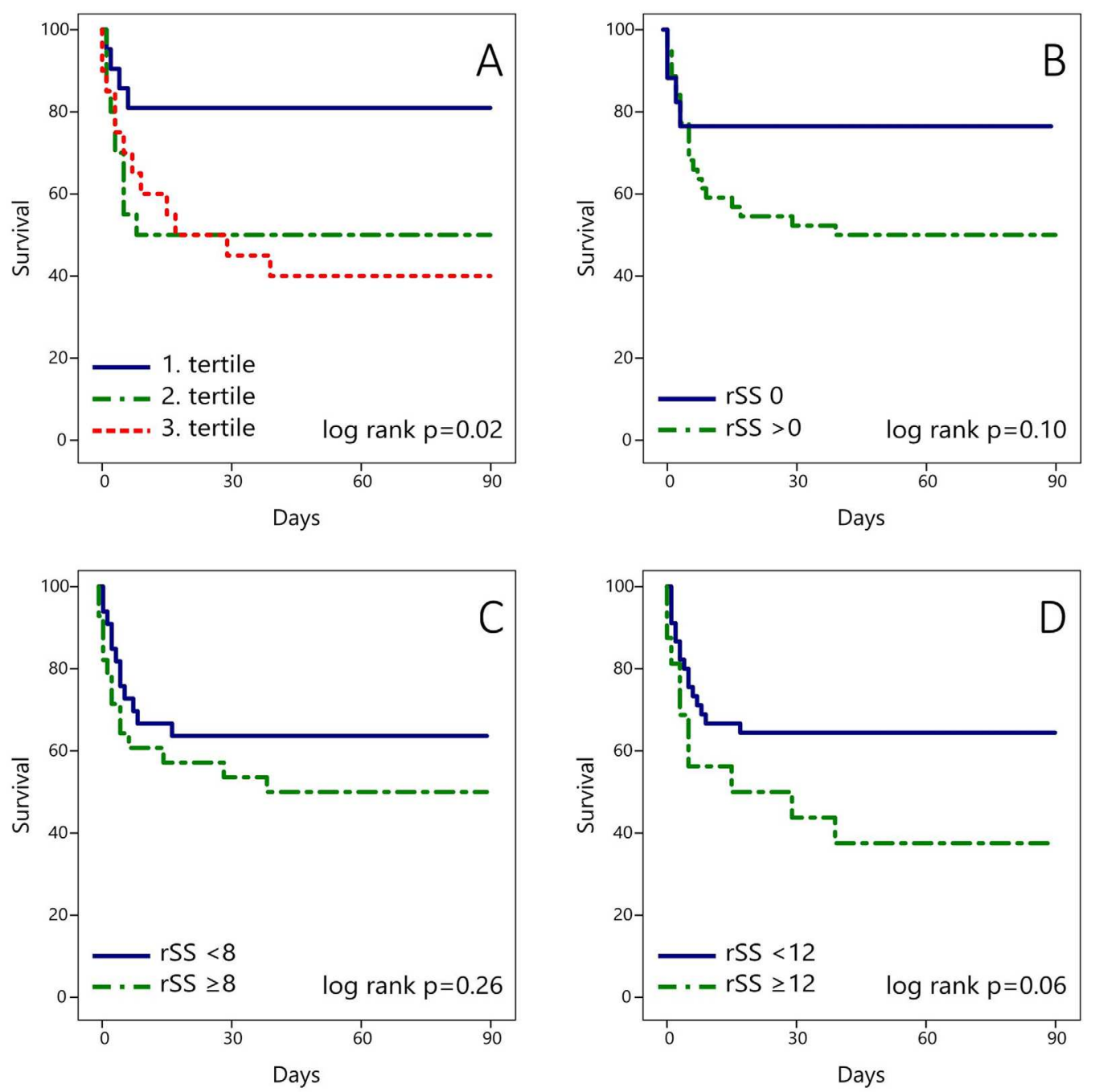

FIGURE 2 Ninety-day survival analysis-A, Kaplan-Meier's survival curves in bSS1 tertiles; B-D, and in different rSS cutoff points [Color figure can be viewed at wileyonlinelibrary.com]

TABLE 2 The Cox survival models with bSS and rSS as continuous variable for 90 -day all-cause mortality

\begin{tabular}{|c|c|c|c|c|c|c|c|c|}
\hline \multicolumn{2}{|l|}{ (A) } & \multirow{2}{*}{$\begin{array}{l}\text { HR } \\
1.04\end{array}$} & \multirow{2}{*}{$\begin{array}{l}95 \% \mathrm{Cl} \\
1.01-1.08\end{array}$} & \multicolumn{2}{|l|}{$P$-value } & \multirow{2}{*}{$\begin{array}{l}\text { HR } \\
1.04\end{array}$} & \multirow{2}{*}{$\begin{array}{l}95 \% \mathrm{Cl} \\
1.00-1.08\end{array}$} & \multirow{2}{*}{$\begin{array}{c}P \text {-value } \\
0.04\end{array}$} \\
\hline Univariable & $b S S^{b}$ & & & 0.02 & $r S S^{\mathrm{b}}$ & & & \\
\hline \multicolumn{9}{|c|}{ Multivariable } \\
\hline & $b S S^{b}$ & 1.06 & $1.01-1.10$ & 0.03 & $r S S^{\mathrm{b}}$ & 1.03 & $0.99-1.08$ & 0.14 \\
\hline & $\mathrm{LVEF}^{\mathrm{c}}$ & 1.80 & $1.22-2.65$ & $<0.01$ & $\mathrm{LVEF}^{\mathrm{c}}$ & 1.87 & $1.31-2.69$ & $<0.01$ \\
\hline & Lactate $^{d}$ & 1.23 & $1.08-1.39$ & $<0.01$ & Lactate $^{d}$ & 1.21 & $1.08-1.36$ & $<0.01$ \\
\hline \multicolumn{9}{|c|}{ Clinical risk scores } \\
\hline & CardShock $^{\mathrm{b}}$ & 1.79 & $1.36-2.34$ & $<0.01$ & CardShock $^{\mathrm{b}}$ & 1.81 & $1.38-2.37$ & $<0.01$ \\
\hline & $b S S^{b}$ & 1.02 & $0.98-1.06$ & 0.29 & $r S S^{\mathrm{b}}$ & 1.02 & $0.98-1.06$ & 0.44 \\
\hline & IAPB-SHOCK II & 2.00 & $1.52-2.62$ & $<0.01$ & IAPB-SHOCK II & 1.86 & $1.42-2.42$ & $<0.01$ \\
\hline & $b S S^{b}$ & 1.05 & $1.01-1.09$ & $<0.01$ & $r S S^{b}$ & 1.03 & $0.99-1.07$ & 0.08 \\
\hline
\end{tabular}

\footnotetext{
a See Section 2 for variables assessed.

${ }^{b}$ Per one point increase.

c Per $10 \%$ decrease.

d Per $1 \mathrm{mmol} / \mathrm{l}$ increase.
} 
TABLE 3 The Cox survival models with rSS as categorical variable with different cutoffs for 90-day all-cause mortality

\begin{tabular}{|c|c|c|c|c|}
\hline & & HR & $95 \% \mathrm{Cl}$ & $P$-value \\
\hline \multicolumn{5}{|l|}{ (A) } \\
\hline \multirow[t]{3}{*}{ Univariable } & $r S S>0$ & 2.34 & $0.81-6.80$ & 0.09 \\
\hline & $\mathrm{rSS} \geq 8$ & 1.55 & $0.72-3.35$ & 0.27 \\
\hline & $r S S \geq 12$ & 2.05 & $0.93-4.52$ & 0.08 \\
\hline \multicolumn{5}{|l|}{ Multivariable } \\
\hline \multirow[t]{3}{*}{ Control variables $^{a}$} & LVEF $^{\mathrm{b}}$ & & & $<0.01^{\mathrm{e}}$ \\
\hline & Lactate $^{c}$ & & & $<0.01^{\mathrm{e}}$ \\
\hline & Post-PCI TIMI 3 & & & $<0.01^{\mathrm{e}}$ \\
\hline \multirow[t]{3}{*}{ and either } & (1) $r S S>0$ & 2.46 & $0.83-7.29$ & 0.11 \\
\hline & (2) $\mathrm{rSS} \geq 8$ & 1.27 & $0.55-2.94$ & 0.58 \\
\hline & (3) $r S S \geq 12$ & 1.54 & $0.65-3.66$ & 0.33 \\
\hline \multicolumn{5}{|l|}{ Clinical risk scores } \\
\hline & CardShock $^{d}$ & & & $<0.01^{\mathrm{e}}$ \\
\hline \multirow[t]{4}{*}{ and either } & (1) $r S S>0$ & 2.41 & $0.80-7.26$ & 0.12 \\
\hline & (2) $r S S \geq 8$ & 1.05 & $0.48-2.31$ & 0.91 \\
\hline & (3) $r S S \geq 12$ & 1.26 & $0.55-2.88$ & 0.58 \\
\hline & IABP-SHOCK II & & & $<0.01^{\mathrm{e}}$ \\
\hline \multirow[t]{4}{*}{ and either } & (1) $r S S>0$ & 2.05 & $0.70-6.00$ & 0.19 \\
\hline & (2) $r S S \geq 8$ & 1.36 & $0.62-2.99$ & 0.45 \\
\hline & (3) $r S S \geq 12$ & 1.53 & $0.67-3.49$ & 0.31 \\
\hline & GRACE $^{d}$ & & & $0.01^{\mathrm{e}}$ \\
\hline \multirow[t]{3}{*}{ and either } & (1) $r S S>0$ & 1.83 & $0.62-5.43$ & 0.28 \\
\hline & (2) $r S S \geq 8$ & 1.44 & $0.67-3.11$ & 0.36 \\
\hline & (3) $r S S \geq 12$ & 1.71 & $0.77-3.80$ & 0.19 \\
\hline
\end{tabular}

a See Section 2 for variables assessed.

b Per $10 \%$ decrease.

c Per one $\mathrm{mmol} / \mathrm{l}$ increase.

d Per one point increase.

e $P$-values calculated and valid for all categorical rSS multivariable models. in this study is quite small, meaning that the mortality analyses do have to be assessed with caution.

Regarding the analysis of rSS, some of the patients were diagnosed with CS only after coronary angiography, when optimally, all patients should have presented with CS before revascularization. However, this study reflects real life practice, since CS most often develops during hospitalization, ${ }^{25}$ and in the current era of immediate revascularization in STEMI, it is evident that some patients develop CS only after the procedure. In addition, most study patients who developed CS after revascularization, did it during the first hours after angiography, suggesting that the pathophysiologic changes of CS had probably started even before coronary angiography, and some clinical signs of CS may already have been present. Therefore, we believe our results can be useful in the clinical practice.

The distinctive improvement from bSS to rSS implies successful revascularization. However, the rate of complete revascularization (rSS 0) was lower than in prior studies ${ }^{13,20,21,26}$ and it was reached most often in patients with the lowest bSS. As complete revascularization is not always achievable in acute setting, previous studies have examined the concept of reasonable incomplete revascularization in ACS, but not in CS. Previously, the cutoff has been most often set at rSS $8,{ }^{14,20,21,27}$ or 12 points. ${ }^{9,12}$ In contrast to the earlier studies in non-CS populations, we found no prognostic effect whether we used the rSS cutoff of 8 or 12 points.

We could not demonstrate any predictive value of rSS in STEMIrelated CS. In previous studies, rSS independently predicted mortality in STEMI. ${ }^{12,14,27}$ Recent CULPRIT-SHOCK trial showed worse survival in $\mathrm{CS}$ in patients treated with multivessel $\mathrm{PCl}$ in comparison with revascularization of culprit artery only, ${ }^{8}$ and the earlier studies have shown similar results ${ }^{28}$ or they have found no survival benefit. ${ }^{29}$ In our study, rSS had only modest association with mortality in univariate analysis, but the prognostic value was outweighed by the clinical variables and the risk scores in adjusted analysis. The difference in the predictive power of rSS probably relates to the difference between

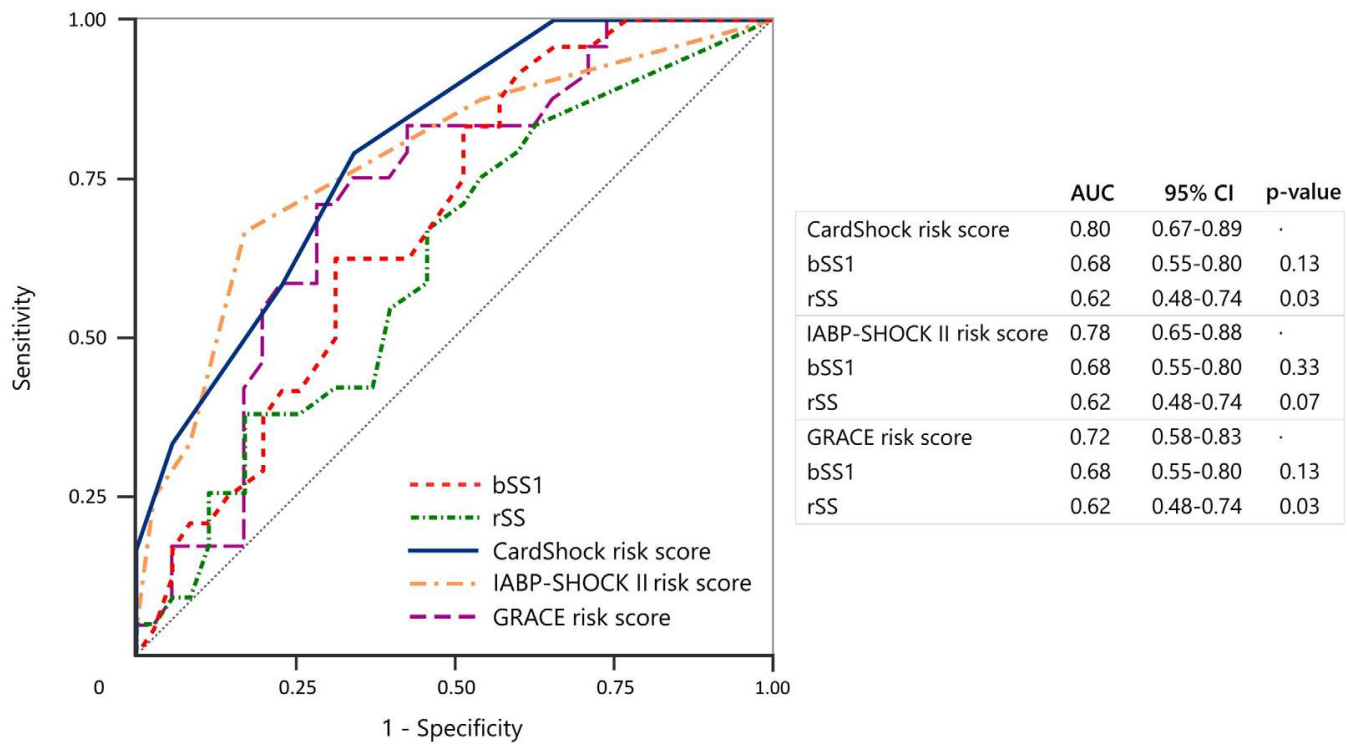

FIGURE 3 The prognostic ability of SYNTAX scores-ROC curves of bSS, rSS, and clinical risk scores [Color figure can be viewed at wileyonlinelibrary.com] 
the severity of clinical condition, i.e., CS and any ACS. The patients in our study were seriously ill: one third of the patients had been resuscitated and over half had CS before PCI. In such circumstances, revascularization is challenging, and treatment targets must include hemodynamic stability, adequate organ perfusion and limiting multiorgan failure. Regarding the timing of CS and the completeness of revascularization, we found that rSS was equal in patients who developed CS before or after angiography. This finding could suggest that less complete revascularization was not the cause of CS in the patients who developed CS after the procedure. However, considering the small sample size of the study, straightforward conclusions are difficult to make regarding the relationship between the failure of revascularization and the cause of the CS. Nevertheless, in line with previous studies, successful revascularization of the IRA, defined as achieving post-PCI TIMI flow 3, was associated with lower mortality. ${ }^{16}$ This may suggest that restoring the blood flow to the IRA is more important than targeting complete revascularization and low rSS.

\section{5 | LIMITATIONS}

Several limitations of our study need to be considered. First, the cohort consists of a limited number of patients, and despite high mortality rates, the number of events in mortality analyses is small. Nevertheless, this is to our knowledge the first, and thus the largest analysis of SS in a cohort of CS patients. Second, interpretation of the angiograms was not centralized, but the SS was analyzed in each hospital by one local experienced cardiologist. However, both were well trained to calculate the SS. Third, the CardShock risk score was developed from the original CardShock cohort including also the patients in the current study, which could affect the analysis of the prognostic value of bSS and rSS over the CardShock risk score. However, the added value of SS was limited also when comparing to two other risk scores.

\section{6 | CONCLUSION}

In STEMI-related CS, the additive value of bSS and rSS over clinical assessment and risk scores is limited. Our results support that in STEMI-related CS, restoring the blood flow to the IRA is essential, and more important than opting for the complete revascularization with $\mathrm{PCl}$.

\section{ACKNOWLEDGMENT}

The authors would like to acknowledge the CardShock study investigators and the GREAT network.

\section{CONFLICT OF INTEREST}

The authors have no conflicts of interest to declare.

\section{REFERENCES}

1. Serruys PW, Onuma Y, Garg S, et al. Assessment of the SYNTAX score in the syntax study. Eurolntervention. 2009;5:50-56.

2. Garg S, Sarno G, Serruys PW, et al. Prediction of 1-year clinical outcomes using the SYNTAX score in patients with acute ST-segment elevation myocardial infarction undergoing primary percutaneous coronary intervention. JACC Cardiovasc Interv. 2011;4:66-75.

3. Palmerini T, Genereux P, Caixeta A, et al. Prognostic value of the SYNTAX score in patients with acute coronary syndromes undergoing percutaneous coronary intervention: Analysis from the ACUITY (acute catheterization and urgent intervention triage StrategY) trial. J Am Coll Cardiol. 2011;57:2389-2397.

4. Onuk T, Gungor B, Ipek G, Karatas MB, Canga Y, Akyuz S, Cam N. Comparison of long-term prognostic value of baseline SYNTAX and clinical SYNTAX scores in ST-segment elevation myocardial infarction patients with multivessel disease. Coron Artery Dis. 2016;27: 311-318.

5. Magro M, Nauta S, Simsek C, et al. Value of the SYNTAX score in patients treated by primary percutaneous coronary intervention for acute ST-elevation myocardial infarction: The MI SYNTAX score study. Am Heart J. 2011;161:771-781.

6. Magro M, Räber L, Heg D, et al. The MI SYNTAX score for risk stratification in patients undergoing primary percutaneous coronary intervention for treatment of acute myocardial infarction: A substudy of the COMFORTABLE AMI trial. Int J Cardiol. 2014;175:314-322.

7. Levine GN, Bates ER, Blankenship JC, et al. 2011 ACCF/AHA/SCAI guideline for percutaneous coronary intervention. A report of the American College of Cardiology Foundation/American Heart Association task force on practice guidelines and the Society for Cardiovascular Angiography and Interventions. J Am Coll Cardiol. 2011;58:e44-e122.

8. Thiele H, Akin I, Sandri M, et al. PCl strategies in patients with acute myocardial infarction and cardiogenic shock. N Engl J Med. 2017;377: 2419-2432.

9. Jang WJ, Yang JH, Song YB, et al. Clinical implications of residual SYNTAX score after percutaneous coronary intervention in patients with chronic total occlusion and multivessel coronary artery disease: A comparison with coronary artery bypass grafting. Eurolntervention. 2017;13:97-105.

10. Garg S, Sarno G, Girasis C, et al. A patient-level pooled analysis assessing the impact of the SYNTAX (synergy between percutaneous coronary intervention with Taxus and cardiac surgery) score on 1-year clinical outcomes in 6,508 patients enrolled in contemporary coronary stent trials. JACC Cardiovasc Interv. 2011;4:645-653.

11. Witberg G, Lavi I, Assali A, Vaknin-Assa H, Lev E, Kornowski R. The incremental impact of residual SYNTAX score on long-term clinical outcomes in patients with multivessel coronary artery disease treated by percutaneous coronary interventions. Catheter Cardiovasc Interv. 2015;86:3-10.

12. Singbal Y, Fryer M, Garrahy P, Lim R. Baseline and residual SYNTAX score in predicting outcomes after acute infarct angioplasty. Eurolntervention. 2017;12:1995-2000.

13. Park KW, Kang J, Kang S-H, et al. The impact of residual coronary lesions on clinical outcomes after percutaneous coronary intervention: Residual SYNTAX score after percutaneous coronary intervention in patients from the efficacy of Xience/Promus versus cypher in rEducing late loss after stENTing (EXCELLENT) registry. Am Heart J. 2014;167:384-392.e5.

14. Khan R, Al-Hawwas M, Hatem R, Azzalini L, Fortier A, Joliecoeur E, Ly $\mathrm{H}$. Prognostic impact of the residual SYNTAX score on in-hospital outcomes in patients undergoing primary percutaneous coronary intervention. Catheter Cardiovasc Interv. 2016;88:740-747.

15. Harjola VP, Lassus J, Sionis A, Køber L, Tarvasmäki T, Spinar J, Parissis J, Banaszewski M, Silva-Cardoso J, Carubelli V, di Somma S, Tolppanen H, Zeymer U, Thiele H, Nieminen MS, Mebazaa A, CardShock Study Investigators, GREAT network. Clinical picture and risk prediction of short-term mortality in cardiogenic shock. Eur J Heart Fail 2015;17:501-509.

16. Poss J, Koster J, Fuernau G, Eitel I, de Waha S, Ouarrak T, Desch S. Risk stratification for patients in cardiogenic shock after acute myocardial infarction. J Am Coll Cardiol. 2017;69:1913-1920. 
17. Fox KAA, Dabbous OH, Goldberg RJ, et al. Prediction of risk of death and myocardial infarction in the six months after presentation with acute coronary syndrome: Prospective multinational observational study (GRACE). BMJ. 2006;333:1091.

18. Thygesen K, Alpert JS, Jaffe AS, et al. Third universal definition of myocardial infarction. Eur Heart J. 2012;33:2551-2567.

19. Sianos G, Morel MA, Kappetein AP, et al. The SYNTAX score: An angiographic tool grading the complexity of coronary artery disease. Eurolntervention. 2005;1:219-227.

20. Genereux P, Palmerini T, Caixeta A, Rosner G, Green P, Dressler O, Stone GW. Quantification and impact of untreated coronary artery disease after percutaneous coronary intervention: The residual SYNTAX (synergy between PCl with Taxus and cardiac surgery) score. J Am Coll Cardiol. 2012;59:2165-2174.

21. Farooq V, Serruys PW, Bourantas CV, et al. Quantification of incomplete revascularization and its association with five-year mortality in the synergy between percutaneous coronary intervention with taxus and cardiac surgery (SYNTAX) trial validation of the residual SYNTAX score. Circulation. 2013;128:141-151.

22. Serruys PW, Morice M-C, Kappetein AP, et al. Percutaneous coronary intervention versus coronary-artery bypass grafting for severe coronary artery disease. N Engl J Med. 2009;360:961-972.

23. Lin MJ, Chen CY, Lin HD, Wu HP. Prognostic analysis for cardiogenic shock in patients with acute myocardial infarction receiving percutaneous coronary intervention. Biomed Res Int. 2017;2017:8530539.

24. Brkovic V, Dobric M, Beleslin B, et al. Additive prognostic value of the SYNTAX score over GRACE, TIMI, ZWOLLE, CADILLAC and PAMI risk scores in patients with acute ST-segment elevation myocardial infarction treated by primary percutaneous coronary intervention. Int J Cardiovasc Imag. 2013;29:1215-1228.

25. Jeger RV, Radovanovic D, Hunziker PR, et al. Ten-year trends in the incidence and treatment of cardiogenic shock. Ann Intern Med. 2008; 149:618-626.
26. Malkin CJ, George V, Ghobrial MS, Krishnan A, Siotia A, Raina T, Gunn J. Residual SYNTAX score after PCl for triple vessel coronary artery disease: Quantifying the adverse effect of incomplete revascularisation. Eurolntervention. 2013;8:1286-1295.

27. Braga CG, Cid-Alvarez AB, Dieguez AR, Alvarez BA, Otero DL, Sanchez RO, Gonzalez-Juanatey JR. Prognostic impact of residual SYNTAX score in patients with ST-elevation myocardial infarction and multivessel disease: Analysis of an 8-year all-comers registry. Int $J$ Cardiol. 2017;243:21-26.

28. Zeymer $\mathrm{U}$, Hochadel $\mathrm{M}$, Thiele $\mathrm{H}$, et al. Immediate multivessel percutaneous coronary intervention versus culprit lesion intervention in patients with acute myocardial infarction complicated by cardiogenic shock: Results of the ALKK-PCI registry. Eurolntervention. 2015;11: 280-285.

29. Bauer T, Zeymer U, Hochadel M, et al. Use and outcomes of multivessel percutaneous coronary intervention in patients with acute myocardial infarction complicated by cardiogenic shock (from the EHS-PCI registry). Am J Cardiol. 2012;109:941-946.

\section{SUPPORTING INFORMATION}

Additional supporting information may be found online in the Supporting Information section at the end of the article.

How to cite this article: Javanainen T, Sans-Roselló J, Harjola V-P, et al. Prognostic impact of baseline and residual SYNTAX scores in cardiogenic shock. Catheter Cardiovasc Interv. 2019;93:1-8. https://doi.org/10.1002/ccd.27716 